\title{
Single-fraction high-dose-rate brachytherapy using real-time transrectal ultrasound based planning in combination with external beam radiotherapy for prostate cancer: dosimetrics and early clinical results
}

\author{
Olivier Lauche, MD',2, Guila Delouya, MD', Daniel Taussky, MD', Cynthia Menard, MD', Dominic Béliveau-Nadeau, MSc', \\ Yannick Hervieux, MSc', Renée Larouche, MSc', Maroie Barkati, MD'
}

'Département de Radio-Oncologie, Centre hospitalier de l'Université de Montréal - Hôpital Notre-Dame, Montréal, Québec, Canada, 2Département de Radiothérapie-Oncologique, Institut du Cancer de Montpellier, Montpellier, France

\begin{abstract}
Purpose: To validate the feasibility of a single-fraction high-dose-rate brachytherapy (HDRBT) boost for prostate cancer using real-time transrectal ultrasound (TRUS) based planning.

Material and methods: From August 2012 to September 2015, 126 patients underwent a single-fraction HDRBT boost of 15 Gy using real-time TRUS based planning. External beam radiation therapy (EBRT) (37.5 Gy/15 fractions, $44 \mathrm{~Gy} / 22$ fractions, or $45 \mathrm{~Gy} / 25$ fractions) was performed before $(31 \%)$ or after (69\%) HDRBT boost. Genito-urinary (GU) and gastro-intestinal (GI) toxicity were assessed 4 and 12 months after the end of combined treatment using the international prostate symptom score scale (IPSS) and the common terminology criteria for adverse events (CTCAE) v3.0.

Results: All dose-planning objectives were achieved in $90 \%$ of patients. Prostate $\mathrm{D}_{90} \geq 105 \%$ and $\leq 115 \%$ was achieved in $99 \%$ of patients, prostate $V_{150} \leq 40 \%$ in $99 \%$, prostate $V_{200}<11 \%$ in $96 \%$, urethra $D_{10}<120 \%$ for $99 \%$, urethra $\mathrm{V}_{125}=0 \%$ in $100 \%$, and rectal $\mathrm{V}_{75}<1 \mathrm{cc}$ in $93 \%$ of patients. Median IPSS score was 4 at baseline and did not change at 4 and 12 months after combined treatment. No patients developed $\geq$ grade 2 GI toxicity. With a median follow-up of 10 months, only two patients experienced biochemical failure. Among patients who didn't receive ADT, cumulative percentage of patients with PSA $\leq 1 \mathrm{ng} / \mathrm{ml}$ at 4 and 18 months was respectively $23 \%$ and $66 \%$.

Conclusions: Single-fraction HDRBT boost of 15 Gy using real-time TRUS based planning achieves consistently high dosimetry quality. In combination with EBRT, toxicity outcomes appear promising. A longer follow-up is needed
\end{abstract} to assess long-term outcome and toxicities.

J Contemp Brachytherapy 2016; 8, 2: 104-109 DOI: $10.5114 /$ jcb.2016.59216

Key words: brachytherapy, HDR, prostate cancer, single fraction, TRUS.

\section{Purpose}

Dose escalation using high-dose-rate brachytherapy (HDRBT) boost in combination with external beam radiation therapy (EBRT) is a key point in the local and biochemical control of prostate cancer [1,2]. High-dose-rate brachytherapy boost seems to be an effective technique to achieve safe dose escalation for intermediate and high-risk prostate cancer. The postulated low $\alpha / \beta$ ratio of prostate cancer cells is an argument in favour of the use of HDRBT as large doses per fraction are delivered, making it one of the most efficient and convenient intervention of hypofractionated radiation. In combination with external beam radiation therapy (EBRT), reported series using HDRBT boost describe excellent results in the treatment of intermediate and high-risk prostate cancers [3,4,5]. Morton et al. have demonstrated that a single-fraction HDRBT boost of $15 \mathrm{~Gy}$ using computed tomography (CT) based planning was safe and effective [6,7], and this observation awaits independent confirmation. Single fraction HDRBT boost have many advantages compared to fractionated schedules: no risk of catheters displacement between fractions, better convenience and comfort for the patients who undergo only one procedure in an outpatient basis, and less resource intense.

Real-time transrectal ultrasound (TRUS) based planning is a relatively new HDRBT planning technique that seems to have many advantages compared to CT or magnetic resonance imaging (MRI) based planning: the entire process is completed without moving the patient and it allows time, resources, and cost savings $[8,9]$.

Address for correspondence: Maroie Barkati, MD, Département de Radio-Oncologie, Centre hospitalier $\quad$ Received: 24.12 .2015 de l'Université de Montréal - Hôpital Notre-Dame, Montréal, Québec, 1560 Sherbrooke Est, Montréal, Qc, Accepted: 13.03.2016 H2L 4M1, Canada, phone: +1-514-890-8254, fax: +1-514-412-7537, @ e-mail: maroie.barkati.chum@ssss.gouv.qc.ca Published: 27.04.2016 
We report here dosimetrics and early clinical results of a cohort of patients treated with a single-fraction HDRBT boost of 15 Gy using real-time TRUS based planning in combination with EBRT.

\section{Material and methods}

\section{Patients}

A total of 126 consecutive patients were included in this study between August 2012 and September 2015. Eligible patients had newly diagnosed, intermediate, or high-risk adenocarcinoma of the prostate according to the D'Amico classification [10] with negative distant staging. Abdominal CT and bone scan were not mandatory for intermediate risk patients but were done routinely for higher risk prostate cancer patients. Unfavourable intermediate and high-risk patients also received short or long term androgen deprivation therapy (ADT).

\section{External beam radiation therapy treatment}

External beam radiation therapy dose was $37.5 \mathrm{~Gy}$ in 15 fractions of 2.5 Gy each (prostate and seminal vesicles) for intermediate-risk prostate cancer, and $44 \mathrm{~Gy}$ or $45 \mathrm{~Gy}$ in 22 or 25 fractions (whole pelvis) for high-risk prostate cancers. The clinical target volume (CTV) was defined as the prostate and the proximal first third of the seminal vesicles. For unfavourable intermediate or high-risk patients, pelvic lymph node regions were also included in the CTV (CTV nodal). These include bilateral obturator, bilateral internal, and external iliac and pre-sacral lymph node regions. The planning target volume (PTV) was the CTV plus a $7 \mathrm{~mm}$ margin when fiducial markers were used, or a $10 \mathrm{~mm}$ margin when there were no fiducial markers. In that case, daily soft tissue imaging was performed. For high-risk patients, the PTV around the pelvic lymph node regions was the CTV nodal plus a $7 \mathrm{~mm}$ margin.

External beam radiation therapy was delivered using an intensity modulated radiation therapy (IMRT) technique. Either static IMRT or volumetric modulated arc therapy (VMAT) was used.

\section{High-dose-rate brachytherapy boost treatment}

High-dose-rate brachytherapy boost is delivered either before or after EBRT, depending on the availability of the brachytherapy suite. When ADT is indicated, brachytherapy is delivered 2 months after the initiation of ADT and before EBRT.

High-dose-rate brachytherapy boost is performed in a shielded brachytherapy suite. It is performed under general anaesthesia, which eliminates movements during the procedure compared to spinal anaesthesia. Prophylactic antibiotics are given, usually cephalexine 2 grams intravenously. Patients are positioned in the dorsal lithotomy position and a urinary catheter is inserted. The TRUS probe (flex focus, BK medical ${ }^{\circledR}$, Denmark) attached to a stepper is inserted, and probe is positioned so that the urethra is at the center of the template grid on the ultrasound screen. A template is attached to the ultrasound stepper, through which flexible catheters (Proguide 6F Sharp, length $=240 \mathrm{~mm}$ ) are inserted to the middle of the prostate under TRUS guidance in the axial mode (Figure 1A). Generally, catheters are inserted from the periphery to the center and from the anterior to the posterior border of the prostate. Caution is needed to avoid acoustic shadowing that may create missing catheters. Then, the sagittal mode is selected and catheters are pushed into the prostate to the bladder neck to ensure a good coverage of the prostate base (Figure 1B). To know the exact position of the needle tip, a measurement of the needle lengths protruding beyond the template is performed (Figure 1C) [11]. Once catheters are well positioned, three-dimensional TRUS images are acquired and prostate, anterior rectal wall and urethra are delineated by the radiation oncologist (Figure 1D). Catheters are then reconstructed by a trained medical physicist (Figure 1E). Treatment plans are created and optimized using V4.2 Oncentra Prostate (Elekta ${ }^{\circledR}$, Stockholm, Sweden) treatment planning system (Figure $1 \mathrm{~F}$ ). Dose prescription is $15 \mathrm{~Gy}$ in 1 fraction to the whole prostate with no additional margins. Dose objectives used for treatment planning are those published by Morton et al. [6,7] and are summarized in Table 1.

The catheters are then connected to the afterloader (Microselectron HDR V2, Elekta ${ }^{\circledR}$, Stockholm, Sweden) using transfer tubes and treatment is delivered with the TRUS probe and urinary catheter still in place, without moving the patient. After treatment delivery, fiducial markers are inserted for image-guided radiation therapy if EBRT is to follow HDRBT boost. Total time is about 2 hours [8]. Patient is ready for discharge few hours after the procedure.

\section{Follow-up}

Patients were followed one and four months after the treatment and then every 6 months with symptom assessments, a digital rectal exam if indicated and a prostate specific antigen (PSA) level. Gastro-intestinal (GI) toxicity was scored with common terminology criteria for adverse events (CTCAE) v 3.0 and genito-urinary (GU) toxicity with international prostate symptom score (IPSS). In this study, we report biochemical failure results and toxicity at 4 and 12 months after completion of the entire course of HDRBT boost and EBRT. Because a longer follow-up is needed to assess sexual dysfunction, and due to the important number of patients who received ADT, this toxicity was not assessed. Biochemical failure was defined as PSA nadir plus $2 \mathrm{ng} / \mathrm{ml}$ [12].

\section{Statistical analysis}

Dosimetric data and toxicity are reported using summary descriptive statistics. IPSS results were compared with Wilcoxon rank sum test using SPSS 17.0 for Windows (IBM SPSS, Chicago, IL, USA).

\section{Results}

\section{Patient and treatment characteristics}

Patient and treatment characteristics are reported in Table 2 . The majority of patients were classified as inter- 

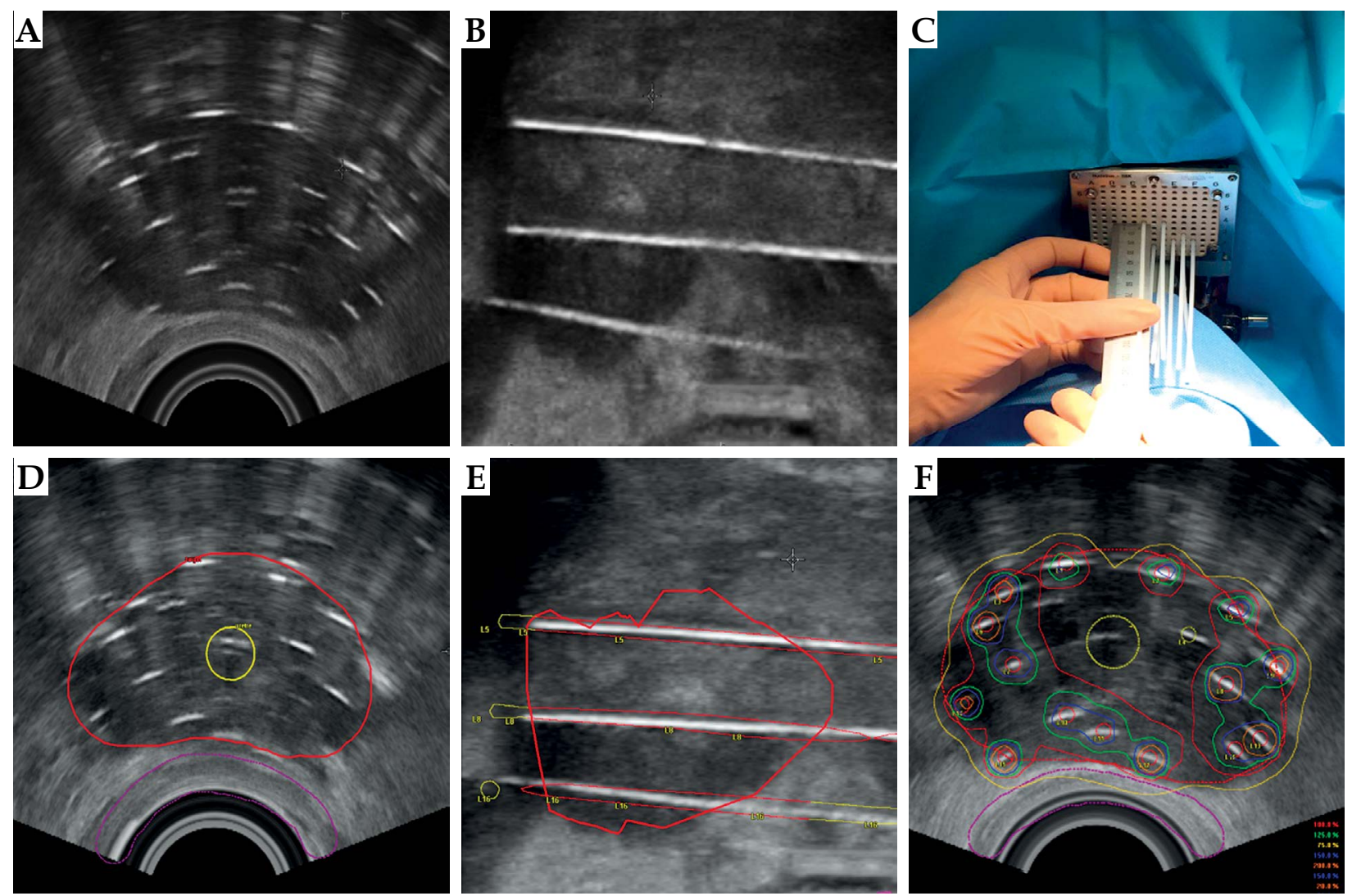

Fig. 1. Transrectal ultrasound-based planning. A) Flexible catheters are inserted to the middle of the prostate under TRUS guidance in the axial mode. B) Catheters are pushed into the prostate to the bladder neck to ensure a good coverage of the prostate base. C) To identify the exact position of the needle tip, a measurement of the needle lengths protruding beyond the template is performed. D) Three-dimensional TRUS images are acquired and prostate, anterior rectal wall and urethra are delineated. E) Catheters are then reconstructed by a trained medical physicist under the physician's supervision. F) Plans are created and optimized using V4.2 Oncentra Prostate (Elekta ${ }^{\circledR}$, Stockholm, Sweden) treatment planning system

Table 1. Dose objectives used for inverse planning

\begin{tabular}{lc} 
Prostate & $105 \% \leq \mathrm{D}_{90} \leq 115 \%$ \\
\cline { 2 - 2 } & $\mathrm{V}_{150} \leq 40 \%$ \\
\hline Urethra & $\mathrm{V}_{200}<11 \%$ \\
\cline { 2 - 2 } & $\mathrm{D}_{10}<120 \%$ \\
\hline Rectum & $\mathrm{V}_{150}=\mathrm{V}_{125}=0$ \\
\cline { 2 - 2 } & $\mathrm{V}_{75}<1 \mathrm{cc}$ \\
\hline & $\mathrm{V}_{100}=0$
\end{tabular}

$V_{75}, V_{100}, V_{125}, V_{150}$ and $V_{200}$ - volume treated to at least $75 \%, 100 \%, 125 \%$, $150 \%$, and $200 \%$ of the prescribed dose, $D_{10}$ and $D_{90}-$ minimal dose received by $10 \%$ and $90 \%$ of the most irradiated volume

mediate risk according to $\mathrm{D}^{\prime}$ Amico classification (81\%) [10]. Thirty-one percent of patients received whole pelvic EBRT (44 Gy in 22 fractions or 45 Gy in 25 fractions). Twenty-eight percent of patients received ADT.

\section{Dosimetric results}

Dosimetric results of HDRBT boost using real-time TRUS based planning are summarized in Table 3. All dose planning objectives were achieved in $90 \%$ of pa- tients. Prostate $D_{90} \geq 105 \%$ but $\leq 115 \%$ was achieved for $99 \%$ of patients, prostate $V_{150} \leq 40 \%$ for $99 \%$, and prostate $\mathrm{V}_{200}<11 \%$ for $96 \%$. $\mathrm{D}_{10}$ urethra $<120 \%$ was achieved for $99 \%$ of patients and $V_{125}=0 \%$ for all patients. Rectum $\mathrm{V}_{75}<1$ cc was achieved for $93 \%$ of patients.

\section{Early toxicity and prostate specific antigen results}

With a median (IQR) follow-up of 10 months (4-18), only 2 patients $(1.9 \%)$ had biochemical failure. Among these 2 patients, one was at high-risk and refused ADT concomitant with this treatment. For patients who didn't receive ADT, median PSA (IQR) was $1.5 \mathrm{ng} / \mathrm{ml}(1.1-2.6)$ at 4 months after combined treatment, $0.9 \mathrm{ng} / \mathrm{ml}(0.7-$ $1.3)$ at 12 months and $0.6 \mathrm{ng} / \mathrm{ml}(0.3-1.4)$ at 18 months (data available for 52, 22, and 15 patients, respectively). Cumulative percentage of available patients with PSA $\leq 1 \mathrm{ng} / \mathrm{ml}$ at 4,12 , and 18 months was respectively $23 \%$, $54 \%$, and $66 \%$. Cumulative percentage of available patients with PSA $\leq 0.5 \mathrm{ng} / \mathrm{ml}$ at 4,12 , and 18 months was $15 \%, 13 \%$, and $46 \%$, respectively.

International Prostate Symptom Score (IPSS) was available for 79 patients at 4 months and for 58 patients at 12 months. There was no statistical differences $(p=0.22)$ between IPSS scores (IQR) at baseline (4 [1-7]) and at 4 months 
Table 2. Patients and treatment characteristics

\begin{tabular}{|c|c|}
\hline Data & Results, $n, \%$ \\
\hline Median age (IQR) & $67(62-71)$ \\
\hline \multicolumn{2}{|l|}{ Tstage } \\
\hline $\mathrm{T} 1-2$ & $98 \%$ \\
\hline $\mathrm{T3}$ & $2 \%$ \\
\hline \multicolumn{2}{|l|}{ Gleason score } \\
\hline 6 & $4 \%$ \\
\hline 7 & $78 \%$ \\
\hline $8-9$ & $18 \%$ \\
\hline Median PSA (IQR) & $8.8(6.5-11.3)$ \\
\hline \multicolumn{2}{|l|}{ Risk groups (D’Amico) } \\
\hline Intermediate & $81 \%$ \\
\hline High & $19 \%$ \\
\hline \multicolumn{2}{|l|}{ ADT } \\
\hline No & $72 \%$ \\
\hline Short term (4-6 months) & $21 \%$ \\
\hline Long term (18-36 months) & $7 \%$ \\
\hline \multicolumn{2}{|l|}{ Timing of HDRBT boost } \\
\hline Before EBRT & $31 \%$ \\
\hline After EBRT & $69 \%$ \\
\hline \multicolumn{2}{|l|}{ EBRT dose schedule } \\
\hline 37.5 Gy/15 f & $69 \%$ \\
\hline 44 Gy/22 f or 45 Gy/25 f & $31 \%$ \\
\hline
\end{tabular}

(4 [1-7]) after combined treatment. Again there was no statistical differences $(p=0.79)$ between IPSS score at baseline and at 12 months (4 [1-7]). One patient developed a urinary infection 2 weeks following HDRBT boost. Only 2 patients had grade 1 GI toxicity 4 months after the end of treatment. No patients experienced $\geq$ grade 2 GI toxicity.

\section{Discussion}

There is mounting evidence suggesting that brachytherapy boost in combination with EBRT provides better results compare to EBRT alone [1,2]. Moreover, brachytherapy boost has the convenience to decrease total treatment time leading to decreased traveling time and expenses.

Many centers have published their own experience of HDRBT boost in combination with EBRT, with various fractionation schedules (15 Gy in 3 fractions, 11-22 Gy in 2 fractions, and 12-15 Gy in 1 fraction). All had very good results so that the Groupe Européen de Curiethérapie - European Society Therapy Radiation Oncology (GEC-
Table 3. Dosimetric data

\begin{tabular}{lc} 
Data & Median (IQR) \\
\hline Prostate volume & $40 \mathrm{~mm}^{3}(30-48)$ \\
\hline Number of catheters & $16(15-17)$ \\
\hline Prostate $\mathrm{D}_{90}$ & $109 \mathrm{~Gy}(107-111)$ \\
\hline Prostate $\mathrm{V}_{100}$ & $98 \%(97-99)$ \\
\hline Prostate $\mathrm{V}_{150}$ & $29 \%(27-32)$ \\
\hline Prostate $\mathrm{V}_{200}$ & $8 \%(7-9)$ \\
\hline Urethra $\mathrm{D}_{10}$ & $115 \mathrm{~Gy}(114-118)$ \\
\hline Urethra $\mathrm{V}_{100}$ & $82 \%(77-88)$ \\
\hline Urethra $\mathrm{V}_{125}$ & $0 \%(0-0)$ \\
\hline Urethra $\mathrm{V}_{150}$ & $0 \%(0-0)$ \\
\hline Rectum $\mathrm{V}_{75}$ & $0.6 \mathrm{~mm}^{3}(0.2-1)$ \\
\hline Rectum $\mathrm{V}_{100}$ & $0 \mathrm{~mm}^{3}(0-0)$
\end{tabular}

$V_{75}, V_{100}, V_{125}, V_{150}$, and $V_{200}$ - volume treated to at least $75 \%, 100 \%, 125 \%$, $150 \%$, and $200 \%$ of the prescribed dose, $D_{10}$ and $D_{90}$ - minimal dose received by $10 \%$ and $90 \%$ of the most irradiated volume, $I Q R$-interquartile range

ESTRO) and the American Brachytherapy Society (ABS) do not recommend one fractionation schedule over another $[12,13,14]$. Morton et al. have published their experience of a single-fraction HDRTBTB (15 Gy) in combination with hypofractionated EBRT (37.5 Gy in 15 fractions). The trial accrued 125 patients with intermediate risk prostate cancers. With a median follow-up of 1.14 years, no patients experienced $\geq$ grade 2 late GI or GU toxicity $[6,7]$, and the 5-year biochemical disease-free survival was over 97\% [9]. Published data on the clinical outcomes of HDRBT boost included patients treated with CT or MRI-based planning. Studies have shown the feasibility of ultrasound catheter tip localization compared with CT imaging $[11,15]$. To our knowledge, this is the first study to report early toxicity and PSA results of HDRBT boost using real-time TRUS-based planning, and the second to report dosimetric and clinical results of single-fraction HDRBT boost of $15 \mathrm{~Gy}$. We were able to achieve excellent dosimetric quality across our cohort using real-time TRUS-guided approach with a median prostate $\mathrm{V}_{100}=98 \%$ (97\% in Morton's study [6]), a median prostate $V_{200}=8 \%$ $(10.7 \%)$, median urethra $\mathrm{D}_{10}=115 \%(119 \%)$, and a rectum $\mathrm{V}_{75}=0.6 \mathrm{~mm}^{3}\left(\mathrm{~V}_{80}=0.76 \mathrm{~mm}^{3}\right)$.

This study suggests that this new real time ultrasound based technique is feasible, safe, and effective not only because dose planning objectives can be consistently achieved but no patients experienced $\geq$ grade 2 GI toxicity. Patients recovered their baseline urinary function 4 months after the end of combined treatment and first PSA responses are promising. One of the limits of this study is the short follow-up (10 months) as this real-time planning technique was introduced in our centre in 2012. Also, longer follow-up is required to fully assess biochemical control outcomes.

The advantage of real-time TRUS-based planning compared to CT or MRI-based planning is that the en- 
tire procedure (catheter insertion, contouring, planning, treatment delivery, and catheter removal) is performed in a shielded room without moving the patient, which reduces the risk of catheters displacements. In fact, mean displacements of $11 \mathrm{~mm}$ have been reported when using CT-based planning [16], which if left uncorrected, would result in poor treatment delivery and prostate base underdosage. Furthermore, the entire procedure including treatment delivery takes an average of 2 hours [8] compared to 4-6 hours with CT or MRI-based planning.

Moreover, single fraction HDRBT boost have many advantages compare to fractionated schedules: no risk of catheters displacement between fractions, better convenience for the patients who undergo only one procedure in an outpatient basis, and less resource consuming.

Additionally, HDRBT boost seems to have many advantages compare to brachytherapy boost using permanent seed implants. First, the very short treatment time and the high dose per fraction lead to a greater efficacy according to the radiobiology of prostate cancer cells [17] and also a quicker recovery from acute side effects. Radiation safety is good, with no need for patients to follow special precautions after the treatment. Likewise, there are no issues in handling radioactive sources by the staff. Also, a single radioactive source may deliver treatment to a large number of patients and it can be used for many disease sites, which is cost-effective. High-dose-rate planning provides multiparametric dose optimization and dose sculpting is possible if one wants to use a concomitant boost on the dominant intraprostatic lesion. The dosimetry is known and approved before treatment delivery and consistently provides good target coverage and normal organ sparing. Finally, catheters can be safely implanted outside the prostate capsule and into the seminal vesicles without the risk of seed migration [18].

Finally, it has been shown that dosimetric quality of HDRBT boost using CT-based planning was significantly improved during the learning curve period [19]. Similarly, catheter localization under TRUS guidance and prostate contouring may be sometimes difficult for untrained physicians because of bright echoes [20] and the learning curve when contouring on ultrasound as most radiation oncologists are trained using CT for planning. Consequently, a good training is needed before performing HDRBT boost using real-time TRUS based planning.

\section{Conclusions}

Real-time TRUS-based planning is a very attractive technique to plan and deliver single-fraction HDRBT. This technique allows planning and treatment delivery without moving the patient, which increases accuracy of treatment delivery and makes the treatment more convenient for the patient as well as more efficient in resource utilization. Our early experience suggests that single fraction HDRBT boost of 15 Gy using real-time TRUS based planning is safe and effective. Finally, a longer follow-up is needed to fully assess long-term outcomes and toxicities.

\section{Disclosure}

Authors report no conflict of interest.

\section{References}

1. Sathya JR, Davis IR, Julian JA et al. Randomized trial comparing iridium implant plus external-beam radiation therapy with external-beam radiation therapy alone in node-negative locally advanced cancer of the prostate. J Clin Oncol 2005; 23: 1192-1199.

2. Hoskin PJ, Rojas AM, Bownes PJ et al. Randomized trial of external beam radiotherapy alone or combined with highdose-rate brachytherapy boost for localised prostate cancer. Radiother Oncol 2012; 103: 217-222.

3. Zwahlen DR, Andrianopoulos N, Matheson B et al. Highdose-rate brachytherapy in combination with conformal external beam radiotherapy in the treatment of prostate cancer. Brachytherapy 2010; 9: 27-35.

4. Kaprealian T, Weinberg V, Speight JL et al. High-dose-rate brachytherapy boost for prostate cancer: comparison of two different fractionation schemes. Int J Radiat Oncol Biol Phys 2012; 82: 222-227.

5. Wilder RB, Barme GA, Gilbert RF et al. Preliminary results in prostate cancer patients treated with high-dose-rate brachytherapy and intensity modulated radiation therapy (IMRT) vs. IMRT alone. Brachytherapy 2010; 9: 341-348.

6. Morton GC, Loblaw DA, Sankreacha R et al. Single-fraction high-dose-rate brachytherapy and hypofractionated external beam radiotherapy for men with intermediate-risk prostate cancer: analysis of short- and medium-term toxicity and quality of life. Int J Radiat Oncol Biol Phys 2010; 77: 811-817.

7. Morton GC, Loblaw DA, Chung $\mathrm{H}$ et al. Health-related quality of life after single-fraction high-dose-rate brachytherapy and hypofractionated external beam radiotherapy for prostate cancer. Int J Radiat Oncol Biol Phys 2011; 80: 1299-1305.

8. Morton GC. Prostate high-dose-rate brachytherapy: transrectal ultrasound based planning, a technical note. Pract Radiat Oncol 2015; 5: 238-240.

9. Morton GC. High-dose-rate brachytherapy boost for prostate cancer: rationale and technique. J Contemp Brachytherapy 2014; 6: 323-330.

10. D'Amico AV, Whittington R, Malkowicz SB et al. Biochemical outcome after radical prostatectomy, external beam radiation therapy, or interstitial radiation therapy for clinically localized prostate cancer. JAMA 1998; 11: 969-974.

11. Schmid M, Crook JM, Batchelar D et al. A phantom study to assess accuracy of needle identification in real-time planning of ultrasound-guided high-dose-rate prostate implants. Brachytherapy 2013; 12: 56-64.

12. Roach M, Hanks G, Thames H et al. Defining biochemical failure following radiotherapy with or without hormonal therapy in men with clinically localized prostate cancer: recommendations of the RTOG-ASTRO Phoenix Consensus Conference. Int J Radiat Oncol Biol Phys 2006; 4: 965-974.

13. Yamada Y, Rogers L, Demanes DJ et al. American Brachytherapy Society consensus guidelines for high-dose-rate prostate brachytherapy. Brachytherapy 2012; 1: 20-32.

14. Hoskin PJ, Colombo A, Henry A et al. GEC/ESTRO recommendations on high dose rate afterloading brachytherapy for localised prostate cancer: An update. Radiother Oncol 2013; 3: 325-332.

15. Batchelar D, Gaztañaga M, Schmid M et al. Validation study of ultrasound-based high-dose-rate prostate brachytherapy planning compared with CT-based planning. Brachytherapy 2014; 1: 75-79. 
16. Holly R, Morton GC, Sankreacha R et al. Use of cone-beam imaging to correct for catheter displacement in high doserate prostate brachytherapy. Brachytherapy 2011; 4: 299-305.

17. Hennequin B, Dubray B. Alpha/beta ratio revisited in the era of hypofractionation. Cancer Radiother 2013; 17: 344-348 [Article in French].

18. Yoshioka Y, Suzuki O, Otani Y et al. High-dose-rate brachytherapy as monotherapy for prostate cancer: technique, rationale and perspective. J Contemp Brachytherapy 2014; 1: 91-98.

19. Benhaïm C, Chand M, Gal J et al. Prostate cancer boost using high-dose-rate brachytherapy: impact of the learning curve on the dosimetry. Cancer Radiother 2014; 7: 659-665.

20. Even AJ, Nuver TT, Westendorp $\mathrm{H}$ et al. High-dose-rate prostate brachytherapy based on registered transrectal ultrasound and in-room cone-beam CT images. Brachytherapy 2014; 2 : 128-136. 